\title{
ANALYZING AND PERSONALIZING THE LEARNING PERFORMANCE FOR SPECIAL NEEDS STUDENTS USING MACHINE LEARNING AND DATA ANALYTICS
}

\author{
Aaron $\mathrm{Fei}^{1}$ and $\mathrm{Yu} \mathrm{Sun}{ }^{2}$ \\ ${ }^{1}$ St. Margaret' s Episcopal High School, 31641 La Novia Ave, \\ San Juan Capistrano, CA 92675, USA \\ ${ }^{2}$ California State Polytechnic University, Pomona, CA, \\ 91768, Irvine, CA 92620, USA
}

\begin{abstract}
Recognizing the fact that autistic kids usually have troubles socially and focusing on academic studies, this research attempts to give a more insightful perspective on the ethnic way of helping autistic kids through technologies [4]. The core idea of this paper is to find a way of helping the autistic kids to maximize their potential instead of accommodating this society using assistive tools. Holding the responsibility of sharing the advantages in this society, this application is built to the end of using a general value to connect level of focus to level of reward. This solution is achieved by three steps: Designing a text box with different variables that evaluates focus level, calculating the level of reward based on achievements on the variables, and the game begins with different hardness according to the level of reward. The results show that the designed application increases the focus level of the kids and their willingness to communicate surprisingly.
\end{abstract}

\section{KEYWORDS}

Python, Computer Science, Autism

\section{INTRODUCTION}

There are 1 in 44 children who are struggling with autism in the United States [5]. Autistic kids usually struggle in focusing, communicating, and socializing with others [6]. The goal is to design an application to help autistic kids to focus. Since autistic children are born with a certain level of disadvantage in society, sharing our advantage with them through the present technology is demanded in present days [14]. To this end, this application is attempting to give an intriguing solution to help the autistic kids through games.

Nowadays, there are many assistive technologies for autistic kids [7]. For example, there are augmentative and alternative communication (AAC), non-verbal people to robots built to increase social skills in children on the spectrum, and picture boards. Even though all of them may have their intention of helping autistic kids in a variety of ways, it is also true that there are a few problems with these assistive technologies [8].

Admittedly, augmentative and alternative communication is a high technology tool for autistic kids to use to improve their social performance. It is true that it has the potential that autistic kids David C. Wyld et al. (Eds): CCNET, NLTM, CICS - 2022 
will spend more time in the process of learning how to use it than using it to improve their skills. Also, it is too expensive to produce widely to allow all the autistic to use it.

Secondly, even though non-verbal people can train the autistic kids according to their own personality, it should be heavily doubted by the practicality of this robot for all autistic kids since the huge population.

Last but not least, paint boards may seem to be a solution to the large population of autistic kids, but its problem is that it never treats the autistic kids equally according to their own circumstances. It is obvious that it is incorrect to use one tool to help all of the kids with completely different demands and personality.

My method is to find a way to encourage their focus by giving awards to evoke their internal motivation to strive [9]. Since the level of focus can be evaluated differently under different situations, I define it as the number of characters that the kid enters in a certain amount of time. So, this tool itself should be used for writing. However, this tool is designed as modularized that could eventually link different applications that evaluate focus levels differently and different games according to individual's demand in future development. This makes my tool easily usable to different individuals, which makes it better than a picture board. It is better than AAC since it is easily usable. Moreover, it is cheaper and allows more autistic kids to use it.

I will give this application to a number of different autistic kids as users to let them use it for a while. Then, I will give them a survey that rates certain aspects of it from 1 to 10. After they answer the questions, I will see if the result is significant enough that I can conclude this application will help them in focusing and studying using the reward system.

The rest of the paper is organized as follows: Section 2 gives the details on the challenges that we met during the experiment and designing the sample; Section 3 focuses on the details of our solutions corresponding to the challenges that we mentioned in Section 2; Section 4 presents the relevant details about the experiment we did, following by presenting the related work in Section 5. Finally, Section 6 gives the conclusion remarks, as well as pointing out the future work of this project.

\section{Challenges}

In order to build the project, a few challenges have been identified as follows.

\subsection{Identifying the Problem and the Righteous Way to Solve it}

Going back to the basics of this problem, these children were born with autism. Then, what should be our proper approach to help them? The moral problem is equality or equity. If we help the autistic kids who were born wealthy, then what about those kids who were born normal but poor. Who should we help to achieve real justice? Taking this problem into consideration, I apply the idea from John Rawls that we should bring the exceeding advantage from us to those people who were born demanding help. Instead of forcing the autistic kids to accommodate the normal way of socializing or giving them assistance to help them communicate, which might eventually be considered as deviant to others, I regard designing a tool that allows the autistic kids to use it according to their free will is more vital [10]. 


\subsection{Difference Between Individuals}

Taking an individual's difference into consideration, I recognize the problem that this application should not simply divide human beings into autistic and unautistic because this is not treating each individual as themselves. To this end, I regard a modularized design is vital that allows more kids with different demands in games and focus areas to use. Even though the application itself is not designed with different choices of games and assistance tools for focus, it generalizes the idea of using a general value to connect the reward and assistance tool to approach this problem. This could be used for future research.

\subsection{Games available and reward level}

There are very few attractive games that will provide their codes to the public. To find a game that is both satisfying having a variable that could change accordingly with level of focus and public is not easy. In addition, to determine the level of reward according to focus to make the game not too easy or too hard is a vital process to make the reward attractive.

\section{Solution}

This application is designed in three major steps. They are input, transfer, and reward. This first step is to give the user an interactive surface to ask for their goal. According to this input value, a timer is going to be set. All the inputs will be calculated after the user clicks. Secondly, the user's performance is going to be evaluated by calculating wpm and checking the time requirement. Through comparison between the time requirement that the user inputs initially and wpm's calculation, the reward is going to be determined as negative reward, positive reward, or normal reward. Lastly, different rewards are going to determine the level of hardness for tetris that changes the speed of blocks falling downward.

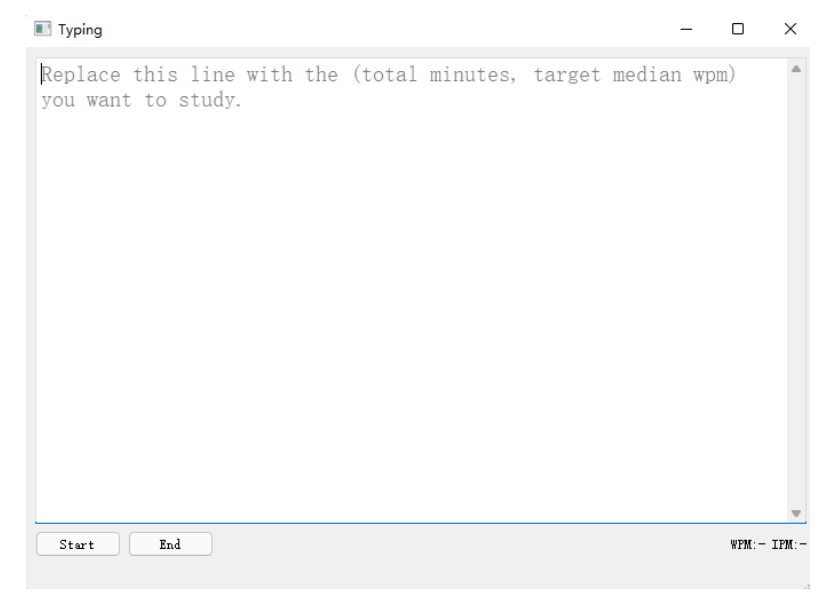

Figure 1. The user interactive surface

The figure above demonstrates the user interactive surface of the text box that constantly updates the user's level of focus in the format of WPM and IPM. Both start and end button were used to confirm the goal of focus level and the concluding time for the application to decide the level of reward. 


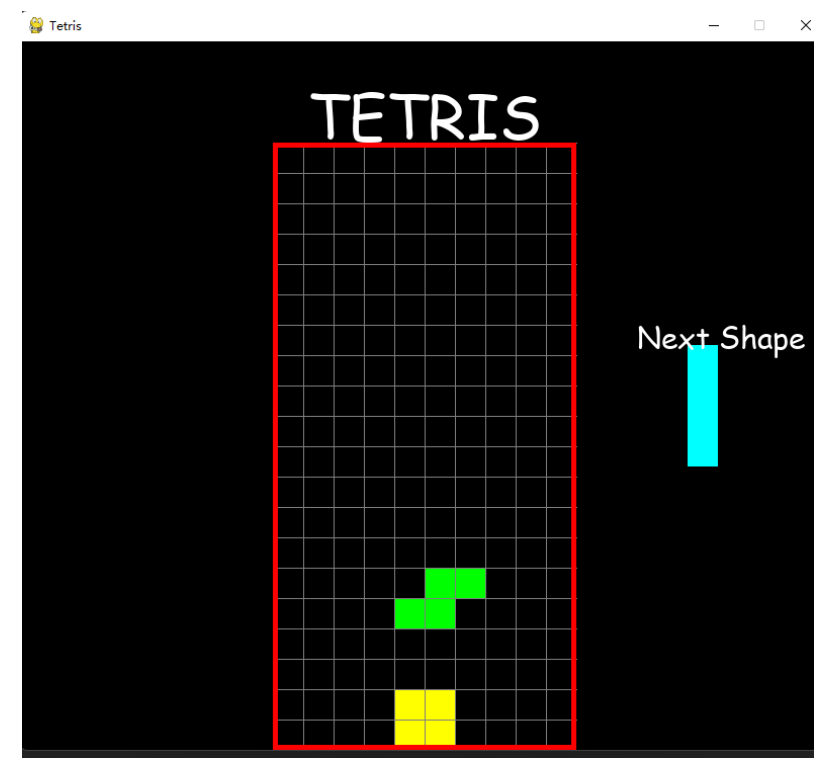

Figure 2. Screenshot of the reward

The above figure demonstrates the reward that is Tetris. The game has almost exactly the same rules as the official game of tetris. To the end of giving users rewards based on their performance during writing, the speed of blocks that were falling has a negative relationship with the achievements on wpm and time requirements.

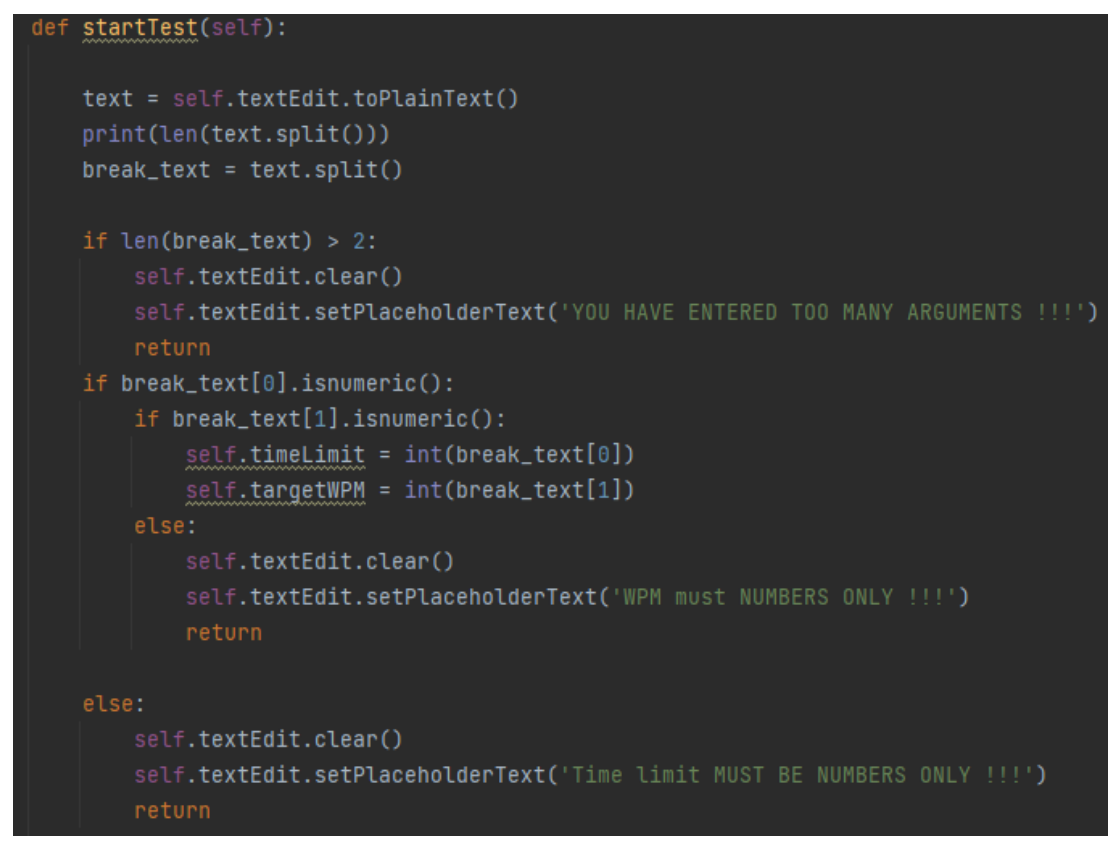

Figure 3. Code of the level of focus

This is a piece of the code that asks the user for the level of focus in a period of literary writing on the computer they are aiming for. Before they start writing, they must input the time limit and wpm goal into the text box; Then, they can click the button to start. In the figure, the user was asked to enter the correct input in order for the application to run successfully by checking if 
there are only two arguments that are both numbers. If not, they must re enter until the application could run successfully.

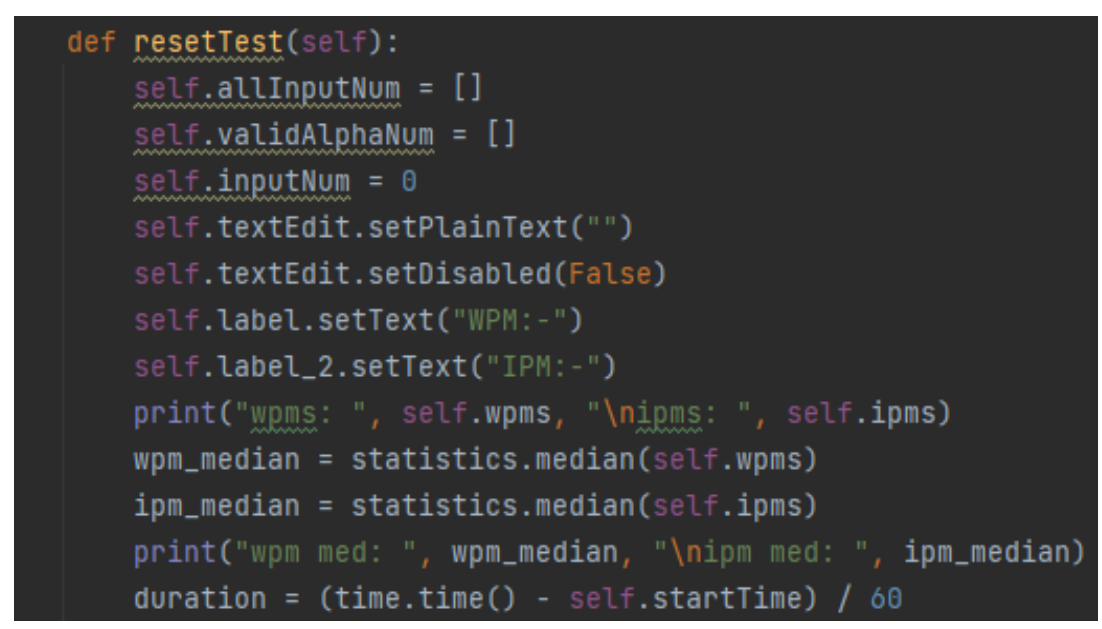

Figure 4. Code of variables

The figure above shows variables that were recorded during the user's input. Wpm is defined as word per minute and Ipm is defined as input per minute. Normally, other applications that assess the level of focus will use the mean of wpms. However, this application uses median to assess a more constant focus. For example, if the user did not write anything in 9 minutes, but copied and past 500 words in the last minute, the mean will demonstrate that he has a 50 wpm, which obviously did not truly demonstrate the user's level of focus. Indeed, a median is used to prevent any false interpretation on the user's focus level in this application.

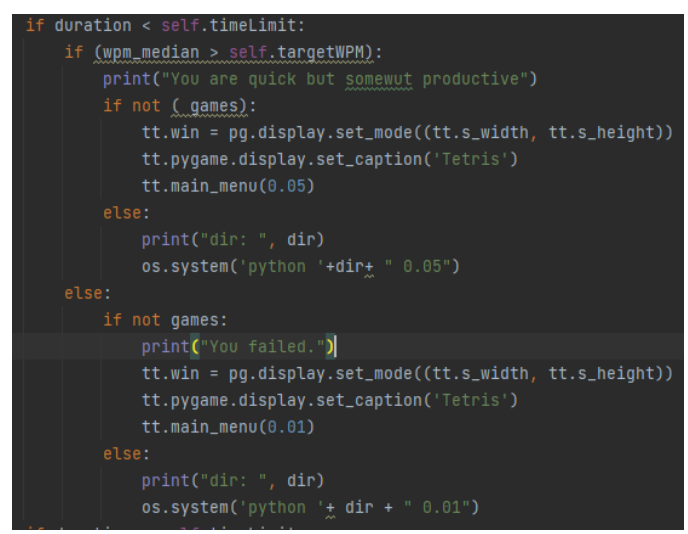

Figure 5 (1). Transfer the variables of level of focus to variables for rewards 


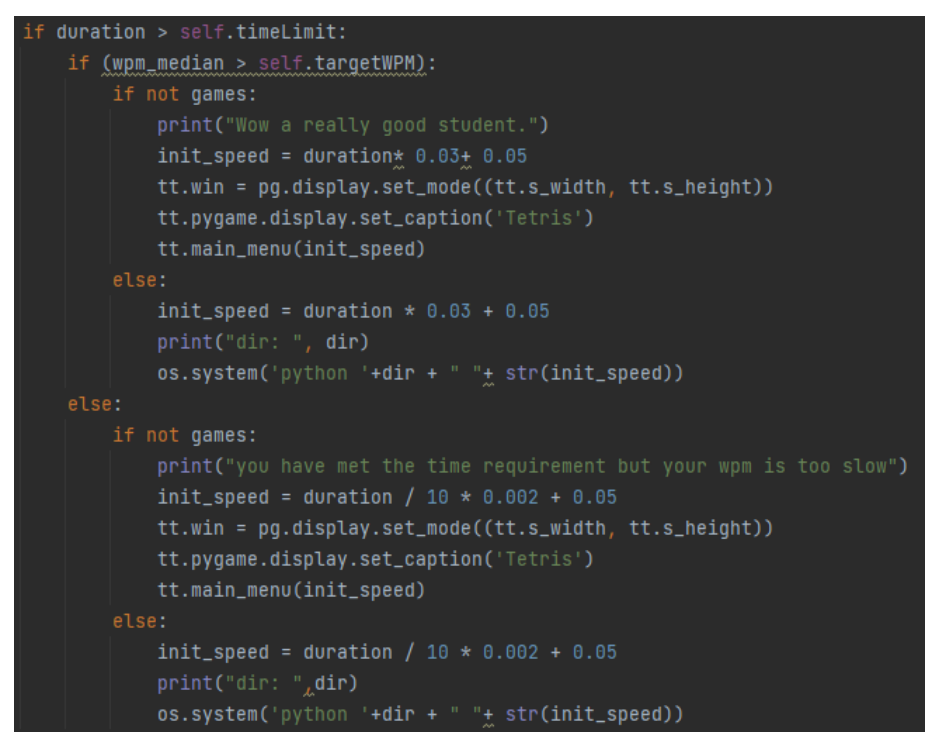

Figure 5 (2). Transfer the variables of level of focus to variables for rewards

The above figures demonstrate statements that transfer the variables of level of focus to variables for rewards. Through the comparison between median wpm and goal wpm, and time written and time requirement goal, the users will have four different results. Giving more weight on the median wpm than time requirement, taking that time requirements could be easily met without achieving productivity, the level of reward will be negative if the median wpm does not meet the goal. If the wpm goal is met, according to the achievement on time requirement, positive and normal rewards will be given accordingly.

\section{EXPERIMENT}

To prove our solution, multiple experiments are run, each with multiple trials. Each experiment alters one part: algorithm parameters, volunteer autistic kids groups from $7-12$, and training data sizes. Through these experiments, we will be able to determine if an algorithm depends on certain factors to produce the best results. Each algorithm is then tested and scored to find the most accurate prediction model. And we will also decide if the solution has an effective influence on autistic kids based on different group-level.

\subsection{Experiment 1}

For the first experiment, the goal is to verify at what age group our application works the best for children with autism. Through sampling 5 groups of children of different ages and letting them use the application from the same time, the result is collected by surveying the parents and counting how many children had significantly improved. Experiments have shown that all children in different age groups show a certain level of interests. Age group 8 and 10 showed the largest interest among all. 


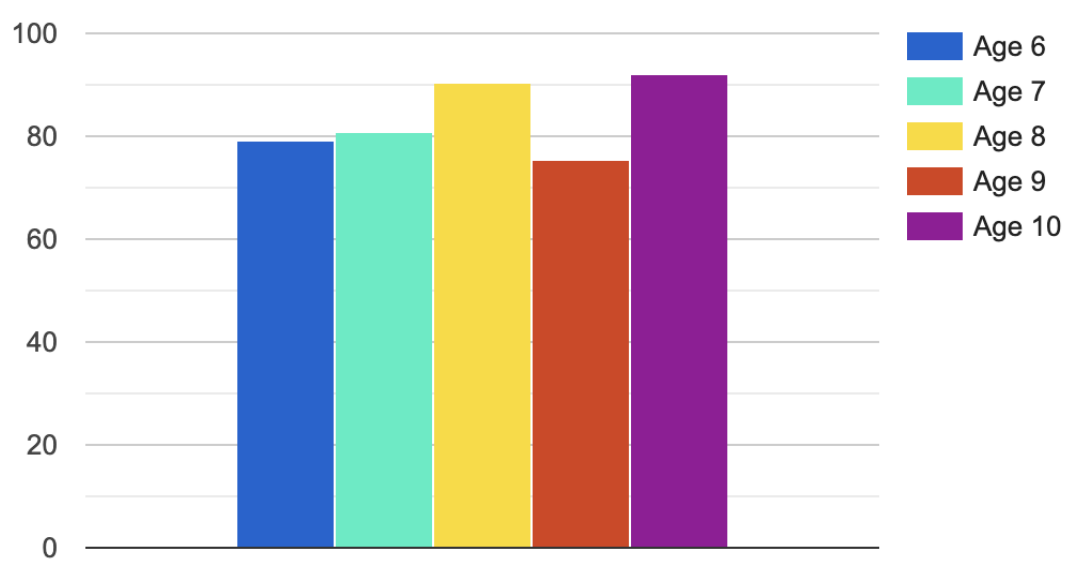

Figure 6. Result of Experiment 1

\subsection{Experiment 2}

The second experiment is to track to what extent the game was able to increase the desire to communicate in children with autism since this is also a part of autistic kid's disadvantages. Surprisingly, this produces a positive result that this paper did not expect initially. By selecting a group of students of the same age and giving a survey to their parents to see if they demonstrate an increase in willingness to communicate after shortly using the application, this experiment demonstrates more than $50 \%$ of kids showed an increase in willingness to communicate.

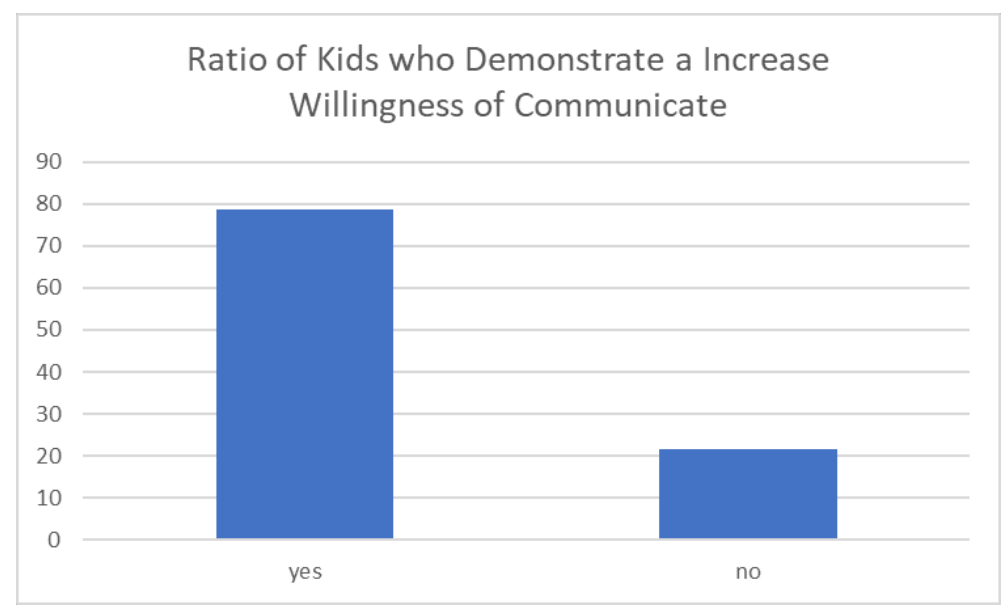

Figure 7. Result of Experiment 2

\subsection{Experiment 3}

The third experiment evaluates the original goal of this paper: the level of increase in focus level. By using the same method of giving surveys to the parents after the last question of assessing the willingness of communication, this question was asked if the kid showed an increase in ability to focus. Indeed, the results demonstrate a positive impact from the application to the autistic kids. 


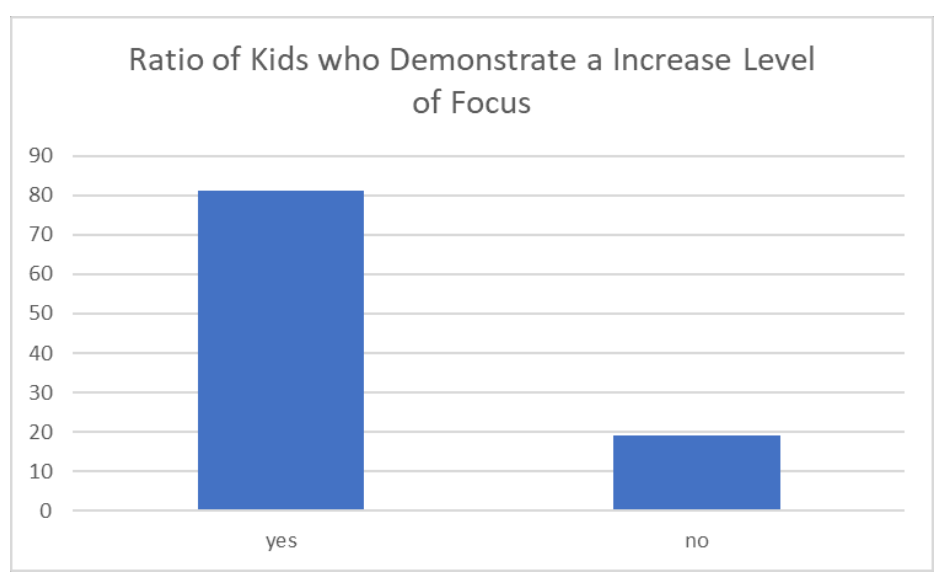

Figure 8. Result of Experiment 3

\section{RELATED WORK}

This paper demonstrates a reevaluation of the previous inventions that were used as assistive technologies for autistic children [1]. After having the result of understanding the application of previous technologies, this paper gives deep insights into limitations of these tools and future directions for these technologies. By interpreting the content while holding the end to develop a better tool for autistic children, this research follows the direction that was summarized from previous tools by combining the advantages and taking the limitations into consideration [15].

This research developed a word processing and organization as an assistance tool to assist students with disabilities to type or increase their performance on computational literature writing [2]. This application provides a similar goal as this paper that is to provide a more equal opportunity for people who were born with disadvantages. The difference between this application and the goal of this paper is that this paper holds the belief that the children could improve their skill under the proper circumstances, but this application could be used more as part of their input process instead of maximizing their own potential. To achieve a better tool for the autistic kids, it might be more helpful for them if this application's design could be incorporated with this paper's design in order to maximize children's potential more efficiently.

This paper analyzes the impact of literature writing on computers to paper and pen writing [3]. Through a demonstration of a positive effect, this analysis inspires the idea of this paper that to design a writing assistive tool for autistic children by strengthening the idea that this application might not only evoke their motivation to write using a reward system, but it may also improve their performance on paper and pen writing, which may eventually help them in social circumstances in reality [12].

\section{CONCLUSIONS}

The purpose of this research is to develop an assistive technology as a tool for autistic kids who lack the ability to focus and socialize to perform better in literature writing and ability to focus [11]. To the end of creating a more equal competing environment for everyone, this paper holds the belief similar to John Rawls that people with advantages have the responsibility to share them with people who don't. Indeed, through a platform utilizing a general variable that connects the basic reward theory and literary writing input surface, this application was formed. After doing experiments and taking other researchers' results into consideration, this research paper did reach its basic goal of assisting the autistic kids. 
However, there are several improvements that could be made to increase the performance of this application regarding the limitations. First, there is a limit on the users for the present design since it only allows users to focus on inputting words, and playing tetris as a reward. However, taking other situations into consideration, this application will not be useful if the autistic kids were given homework to read. Also, tetris may not inspire all autistic kids' internal motivations. Moreover, this application could only help kids with a slight autism who could focus more easily than those with more severe symptoms.

As this paper had mentioned in the beginning, these limitations were already taken into consideration when this research was initialized. The idealized way to approach these limitations was to use a modularized design to reach the goal of individualizing this application for each user. This goal could be reached by developing more tools like linking to specific web pages or as a google extension that assist the autistic kids to focus using other ways. In addition, connection to more games is imperative as well to satisfy different autistic children's demands on games to evoke each of their internal motivation to maximum. For more severe autistic children, further investigation is required to find the way to help them.

\section{REFERENCES}

[1] Knight, V., McKissick, B.R. \& Saunders, A. A Review of Technology-Based Interventions to Teach Academic Skills to Students with Autism Spectrum Disorder. J Autism Dev Disord 43, 2628 - 2648 (2013). https://doi.org/10.1007/s10803-013-1814-y

[2] Montgomery, D. J., \& Marks, L. J. (2006). Using Technology to Build Independence in Writing for Students With Disabilities. Preventing School Failure: Alternative Education for Children and Youth, 50(3), 33-38. doi:10.3200/PSFL.50.3.33-38

[3] Langone, J., Willis, C., Malone, M., Clees, T., \& Koorland, M. (1994). Effects of Computer-Based Word Processing Versus Paper/Pencil Activities on the Paragraph Construction of Elementary Students With Learning Disabilities. Journal of Research on Computing in Education, 27(2), 171-183. doi:10.1080/08886504.1994.10782125

[4] Bleuler, Eugen. "20. Autistic Thinking." Organization and pathology of thought. Columbia University Press, 1951. 399-437.

[5] Speaks, Autism. "What is autism." Retrieved on November 17 (2011): 2011.

[6] National Research Council. Educating children with autism. National Academies Press, 2001.

[7] Cook, Albert M., and Susan M. Hussey. "Assistive technologies." Principles and practice (2002).

[8] Scherer, Marcia J., and Rob Glueckauf. "Assessing the benefits of assistive technologies for activities and participation." Rehabilitation Psychology 50.2 (2005): 132.

[9] Weber, Keith. "The relationship of interest to internal and external motivation." Communication Research Reports 20.4 (2003): 376-383.

[10] Castree, Noel. "Socializing nature: Theory, practice, and politics." Social nature: Theory, practice, and politics (2001): 1-21.

[11] Edyburn, Dave L. "Rethinking assistive technology." Special Education Technology Practice 5.4 (2004): 16-23.

[12] Gómez, Luis Felipe. "Time to socialize: Organizational socialization structures and temporality." The Journal of Business Communication (1973) 46.2 (2009): 179-207.

[13] Weiss, Peter. The investigation. Marion Boyars, 1966.

[14] Howlin, Patricia, et al. Treatment of autistic children. John Wiley \& Sons, 1987.

[15] Rutter, Michael. "The treatment of autistic children." Journal of Child Psychology and Psychiatry 26.2 (1985): 193-214.

(C) 2022 By AIRCC Publishing Corporation. This article is published under the Creative Commons Attribution (CC BY) license. 